\title{
Development of a novel intraarticular injection of diclofenac for the treatment of arthritis: a preclinical study in the rabbit model
}

\author{
Xinyuan Wen ${ }^{1}$, Xiaoqing Huang ${ }^{2}$ and Huosheng $\mathrm{Wu}^{2 \boxminus}$ \\ 1Department of Pain, First Affiliated Hospital, Gannan University, Ganzhou, Jiangxi, 341000, China; 2Department of Pain, Affiliated Hospital of \\ Jinggangshan University, Ji an, Jiangxi, 343000, China
}

Purpose: To develop a novel intraarticular injection of diclofenac for the treatment of arthritis. Method: Diclofenac loaded nanoparticles were prepared by a nanoprecipitation technique using Eudragit L 100 as the polymer and polyvinyl alcohol as the surfactant. The nanoparticles were evaluated for particle size, zeta potential, scanning electron microscopy, drug release, encapsulation efficiency, and loading efficiency studies. The optimized nanoparticulate formulation was developed for intra articular injection. Intraarticulate injection was evaluated for $\mathrm{pH}$, appearance, viscosity, osmolarity and syringability studies. The optimized injection formulation was tested in an arthritic model consisting of 25 rabbits. Result: Nanoprecipitation method was found to be suitable for diclofenac nanoparticles. The shape of the prepared nanoparticles was found to be spherical and devoid of any cracks and crevices. The average particle size of a diclofenac nanoparticle was found to range from $87 \pm 0.47$ to $103 \pm 0.26 \mathrm{~nm}$. The zeta potential of the prepared nanoparticles was found to be in the range of $0.598 \pm 0.34$ to $0.826 \pm 0.25 \mathrm{mV}$. The encapsulation efficiency was found to be between $73.45 \%$ to $99.03 \%$, while the drug loading was observed between 10.34 to $35.32 \%$. The percentage drug release at 12 hours was found to range from $73.45 \%$ to $99.03 \%$. Conclusion: The developed intraarticular injection was found to be within the physically and chemically accepted limits. Animals treated with the intra articular injection of diclofenac showed a significant reduction in swelling as compares to the other groups.

Keywords: arthritis, nanoparticles, inflammation, encapsulation efficiency, injection

Received: 24 June, 2020; revised: 05 November, 2020; accepted: 14 December, 2020; available on-line: 22 January, 2021

Ðe-mail: wuhuosheng2020@sina.com

Abbreviations: BSA, Bovine Serum Albumin; DNP's, Diclofenac nanoparticles; EE, Entrapment efficiency; FCA, Freund's complete adjuvant; IA, Intraarticular injections; NASID, non steroidal anti-inflammatory drugs; OA, Osteoarthritis; PBS, phosphate buffer saline; PDI, Polydispersity index; PRP, Platelet rich plasma; PSD, Particle size distribution; PVA, Poly vinyl alcohol; RA, Rheumatoid arthritis

\section{INTRODUCTION}

Arthritis is a chronic autoimmune inflammatory disease covering about $1-2 \%$ population in the developed countries. It is simply inflammation of joints, either single or multiple. Amongst different types of arthritis, osteoarthritis (OA) and rheumatoid arthritis (RA) are the most common (Gibofsky, 2012). Stiffness, pain at joints, and swelling are some of the identification markers for arthritis. The disease is mostly observedin joints of hands, knees, wrists and other parts of the organs for which sometimes loss or impairment of function can happen. Some people may observe redness of skin at the joint surface. Fatigue and/or poor appetite due to decreased RBC count can be also seen. When left untreated, arthritis can cause joint dislocation. A bilateral or symmetrical pattern of progression of disease can be observed in some patients, i. e. either both hands or knees can be affected (Gibofsky, 2012; Trouw et al., 2012). There is a report of some cases where arthritis also affected extra articular sites, such as the mouth, lungs, and eyes. Worsening of symptoms a disease flare was observed in some patients where early intervention and diagnosis was not possible. Proper treatment can ameliorate the symptoms for longer periods of time. Role of the environmental and genetic factors is also important in arthritis. Its treatment aims towards suppressing inflammation and managing its symptoms. Recently, different natural or synthetic disease altering drugs (commonly referred to as the anti-rheumatoid drugs) were used to alter the severity of disease (Malmstrom et al., 2017). As arthritis is a long term physiological condition, work on novel drug delivery techniques must be developed to ensure accurate dosing and disease monitoring (Kim et al., 2014).

Non steroidal anti-inflammatory drugs (NSAID) are one of the best systems to treat both types of arthritis and to relieve pain and inflammation (Nissen et al., 2016). These are highly effective analgesics that offer a good range of benefits in relieving pain. Diclofenac, anNSAID, can be used to treat or relieve the symptoms of arthritis (in both OA and RA) due to its properties to treat moderate pain with effective treatment of ankylosing spondylitis (a type of arthritis which affects joints in the spine). Acute migraine attacks can also be treated with Diclofenac (Van et al., 2015). The drug is also available in various dosage forms, such as the capsules, enteric and extended release tablets, liquid filled capsules and nanoparticulate forms. The drug has been studied extensively in international clinical trials that were successful in proving its efficacy in comparison to other NSAIND (Zhang et al., 2014). The superior safety profile of diclofenac makes it a valuable agent for treating arthritic conditions in long term therapy (Zhang et al., 2014; Liao et al., 2016).

Novel drug delivery systems show some advantages over conventional systems in enhancing and sustaining the delivery of the drug at a targeted site. Multidose drug delivery is possible with this type of systems (Liao et al., 2016). Amongst these nanomedicine or nanoparticulate delivery is comparatively new and still rapidly accepted developing technology (Karuppusamy et al., 2017). Since the past few decades, nanoparticles are the method of 
choice for such particulate delivery formany researchers, which is superior to conventional delivery in terms of higher stability, specificity, drug carrying capacity and controlled drug release for both, hydrophilic and hydrophobic drugs (Couvreur et al., 1995). Apart from particulate carriers their cellular sub-size and most importantly biocompatibility with tissues and biological cells impart the reliability to dosage form in both medical and pharmaceutical field. The polymeric material used here can be of nano scale and serve either as a diagnostic or therapeutic tool to perform targeted drug delivery offering multiple benefits to ameliorate and cure different chronic diseases (Suri et al., 2007). This system shows promising results in many fields, like chemotherapeutic agents, immunotherapeutic agents, and radio-therapeutic agents, etc. Nanoparticles also have shown good results for diagnostic purposes as well.

Intraarticular injections (IA) are practiced as adjacent therapy to the main treatment plan or entirely as a whole and sole treatment to relieve moderate to severe pain in patients with either RA or OA. Once administered, IA can give relief up to 20-24 weeks. Although cost effectiveness is a great concern, the IA injections seem to be a promising way to manage pain associated with the disease and improve the quality of life especially in younger patients (Mirajkar et al., 2018). The current treatment with IA includes delivery of steroids, NSAID, hyaluronic acid and most importantly platelet rich plasma (PRP). Some studies have shown that IA of corticosteroids had been showing clinical results till 6 months and /or even longer with less side effects (Mirajkar et al., 2018; Dong et al., 2012).

\section{MATERIALS AND METHODS}

\section{Materials}

Diclofenac was received as a gift sample from the Guangzhou Baiyunshan Pharmaceutical Co., Ltd., Guangdong, China. Other raw materials, such as Eudragit L 100, polyvinyl alcohol, and acetone were purchased from Merck\& Co, Germany. Poloxamer F 127, HPMC K $100 \mathrm{M}$ and $\mathrm{NaCl}$ were also purchased from Merck \& Co, Germany.

\section{Design of experiment}

For the preparation of diclofenac nanoparticles (DNP's), different ranges and concentrations of polymers were selected using the Design Expert ${ }^{\circledR}$ software (Version: 6. 0. 8). This software gives a statistical prediction of effect of independent variables on dependent variables. The concentration ranges of both, the independent and dependent variables are given in Table 1.
Table 1. Variable and three levels

\begin{tabular}{llll}
\hline $\begin{array}{l}\text { Independent } \\
\text { Variable }\end{array}$ & $\begin{array}{l}\text { Low } \\
\text { level }(-1)\end{array}$ & $\begin{array}{l}\text { Middle } \\
\text { level }(1)\end{array}$ & $\begin{array}{l}\text { High } \\
\text { level }(+1)\end{array}$ \\
\hline \begin{tabular}{l} 
A=Polymer conc. (gm) \\
\hdashline
\end{tabular} & 0.1 & 0.2 & 0.3 \\
\hdashline B=Surfactant conc. (ml) & 5 & 10 & 15 \\
\hline Dependent Variables & & & \\
\hline $\begin{array}{l}\text { Y1=\% Encapsulation } \\
\text { efficiency } \\
\text { Y2=\% Drug release }\end{array}$ & & & \\
\hline
\end{tabular}

\section{Preparation of diclofenac loaded nanoparticles (DNP's)}

Nanoprecipitation technique was used to formulate diclofenac nanoparticles using Eudragit L 100 as the polymer, while poly vinyl alcohol (PVA) was used as the as surfactant. The concentration of polymer and surfactant can be varied, as stated in Table 1. The pre-weighed quantity of polymer (Eudragit L 100) was first made to dissolve in a methanol solution and this solution is referred to as Solution A. Similarly, diclofenac was dissolved in acetone with continuous shaking to form Solution B. Solution A and Solution B were mixed and added drop-wise to the aqueous solution of PVA under continuous stirring speed of $700 \mathrm{rpm}$ for $120 \mathrm{~min}-$ utes (Table 2). The resulting solution was centrifuged at $10000 \mathrm{rpm}$ at $400^{\circ} \mathrm{C}$ for $1 \mathrm{hr}$. The prepared nanoparticles of diclofenac were evaluated for various parameters like surface morphology, encapsulation efficiency, \% yield, in vitro drug release (Karuppusamy et al., 2017).

\section{Preparation of intra articular injection}

The intra articular injection was prepared by using the cold method. Pre-weighed quantity of diclofenac nanoparticles was dissolved in water, with subsequent addition of poloxamer F 127. The whole system was stirred and kept in refrigerator at $4^{\circ} \mathrm{C}$ up to $24 \mathrm{hrs}$ to achieve complete solublization of the drug and to obtain a clear solution. To this solution, other excipients, like HPMC $\mathrm{K} 100 \mathrm{M}$ as a copolymer and $\mathrm{NaCl}$ were added as tonicity modifiers. A continuous stirring was given to the solution to obtain a clear viscous solution. The solution was then aseptically poured into transparent $2 \mathrm{ml}$ ampoules and sterilized by autoclaving at $121^{\circ} \mathrm{C}$ at $15 \mathrm{psi}$ for $20 \mathrm{~min}$ (Egemen et al., 2014).

\section{Evaluation of DNP's}

The surface morphology (size and shape) of DNP'swas evaluated by using transmission electron microscopy (FEI Morgagni 268 D at accelerating voltage of $120 \mathrm{kV}$ ). A drop of diluted sample was used to perform surface analysis, followed by addition of a drop of $1 \%$

Table 2. Formulation of diclofenac nanoparticles

\begin{tabular}{|c|c|c|c|c|c|c|c|c|c|}
\hline Batch code & $\mathrm{F} 1$ & $\mathrm{~F} 2$ & F3 & $\mathrm{F} 4$ & F5 & F6 & F7 & F8 & F9 \\
\hline Diclofenac sodium (mg) & 100 & 100 & 100 & 100 & 100 & 100 & 100 & 100 & 100 \\
\hline Eudragit L 100 (mg) & 0.3 & 0.3 & 0.2 & 0.1 & 0.3 & 0.1 & 0.2 & 0.1 & 0.2 \\
\hline Acetone $(\mathrm{ml})$ & q. s. & q. s. & q. s. & q. s. & q. s. & q. $s$. & q. s. & q. s. & q. $s$. \\
\hline Polyvinyl alcohol (ml) & 10 & 15 & 15 & 5 & 5 & 10 & 5 & 15 & 10 \\
\hline Methanol (ml) & 10 & 10 & 10 & 10 & 10 & 10 & 10 & 10 & 10 \\
\hline Water (ml) & Up to & & & & & & & & \\
\hline
\end{tabular}


phosphotungstic acid on the grid surface. By using this technique, the size and shape of nanoparticles was observed and recorded as a photograph. The zeta potential, i. e. the surface charge of diclofenac nanoparticles, was measured by using the phenomenon of electrophoretic mobility of nanoparticles using the Malvern zeta sizer (version 6.12). If required, samples can be diluted using distilled water or $0.1 \mathrm{mM} \mathrm{KCl}$. The sample was placed in the electrophoretic cell with electrical field applied at $15.2 \mathrm{~V} / \mathrm{cm}$. A particle size distribution (PSD) or polydispersity index (PDI) can be defined as the size distribution obtained from photon correlation spectroscopy analysis. Since this index is a dimensionless number obtained from a correlation function, it ranges from 0.01 (monodispersed particles) to $0.5-0.7$ (poly dispersed or broad size distribution).

The entrapment efficiency (\%E.E.) was determined using UV visible spectrophotometry at $276 \mathrm{~nm}$. The preweighed known amount (W) of diclofenac nanoparticles was dissolved in the solvent and centrifuged at 2000 rpm for 20 minutes. The clear supernatant liquid was taken for UV analysis. The amount of unentrapped drug (w) can be calculated and this value is deducted from the total amount or initial amount.

$\%$ E.E. $=(\mathrm{W}-\mathrm{w} / \mathrm{W}) \times 100$

A loading efficiency (\%L.) differs from entrapment efficiency in extraction of drug content from nanoparticulate formulation using $0.1 \mathrm{M} \mathrm{HCl}$ until the whole sample gets dissolved. Accurately pre-weighed quantity $(25 \mathrm{mg})$ was dissolved in $25 \mathrm{ml}$ of $0.1 \mathrm{M} \mathrm{HCl}$ and filtered using Whatmann filter paper \#22. Dilutions were made as and when required, and readings were taken at $276 \mathrm{~nm}$ using a UV spectrophotometer. The following equation can be used to calculated loading efficiency:

\section{$\% \mathrm{~L} .=(\mathrm{Qn} / \mathrm{Wn}) \times 100$}

In the above equation, $\mathrm{Wn}$ is considered as weight of nanoparticles, while $\mathrm{Qn}$ is the amount of drug present in nanoparticles.

One of the most important parameters to evaluate the release characteristics is in vitro drug releasefrom the formulation. A specific quantity of nanoparticles was weighed and placed in a dialysis bag whose both ends were sealed. This dialysis bag was placed in dissolution media (phosphate buffer $\mathrm{pH}$ 6.8) with traces of $1 \%$ sodium lauryl sulphate. The entire study was conducted using USP type II dissolution test apparatus at $37 \pm 0.5^{\circ} \mathrm{C}$ and $75 \mathrm{rpm}$. About $2 \mathrm{ml}$ of the sample was removed as an aliquot for UV spectrophotometric analysis at predetermined time intervals of 0,15 minutes, 30 minutes, and $1 \mathrm{hr}$. Thus removed aliquots were immediately replaced with the same amount of fresh solvent. The sample was diluted, filtered and analyzed spectrophotometrically at $276 \mathrm{~nm}$.

\section{Evaluation of injection}

The formulation was evaluated for its $\mathrm{pH}$ as well as appearance. A digital $\mathrm{pH}$ meter was used to check $\mathrm{pH}$ of solution. Approximately $10 \mathrm{ml}$ of sample was used to check pH. Visual observations were made to observe appearance of solution. Viscosity of the formulation was measured using Brookfield viscometer RV (spindle no. 21) and T-type helipath spindle. Osmometer (model 3250, version 2.4) was used to check osmolarity of formulation. Syringability is one of the important criteria for injections, as particle size matters the most. It is assured using 18-22 gauge needles. In order to pass the test, the sample must pass through the syringe, the ones which do not, are considered to fail this test.

\section{Selection of animals}

The protocol for experiment was approved by institutional ethical committee and all experiments were performed according to animals' care guideline. Both, the male and female New Zealand White rabbits weighing 2-2.7 kg were selected for carrying out this trial. Experimental animals were left in suspended cages and allowed free access to food and water. A period of about seven days was given to the animals so to make them familiar with environment.

\section{Induction of arthritis}

A total of 25 animals of aforementioned strain were selected. Arthritis was induced in a total of 17 experimental rabbit animals by giving three injections of Bovine Serum Albumin (BSA) in Freund's complete adjuvant (FCA). About $5 \mathrm{mg}$ of BSA was emulsified in $1 \mathrm{ml}$ of FCA which was diluted with $1 \mathrm{ml}$ of phosphate buffer saline (PBS). The second booster dose was administered after 4 weeks as a subcutaneous injection comprising $2.5 \mathrm{mg}$ of BSA in $1 \mathrm{ml}$ of FCA, again diluted with $1 \mathrm{ml}$ PBS. After 5 days, the third and final booster dose was given as intraarticular injection.

\section{Assessment of arthritis}

The joints were decalcified with $5 \%$ formic acid. Sections of bone, cartilage and synovium were prepared and stained with hematoxylin and eosin for proteoglycan content. Synovial inflammation and cartilage degradation were evaluated by blinded histological evaluation of parapatellar synovium and femoral condylar articular cartilage, respectively. The grading of cellular infiltration and swelling was scored from $0-4$ depending on erythema, swelling and cellular infiltration (0, normal; 4, maximum). The score for proteoglycan loss was set from 0 (normal) to 4 (almost total loss of stained proteoglycans) (Armstrong et al., 1981).

\section{RESULTS AND DISCUSSION}

The diclofenac nanoparticles were prepared using the nanoprecipitation method, as it is suitable for poorly water-soluble drugs. It involves precipitation of preformed polymers from organic solvent which diffuses to an aqueous medium using a surfactant. This leads to instantaneous formation of nanoparticles. Semipolar, water miscible solvents, such as the acetone or ethanol, can be used. The shape of the prepared nanoparticles was found to be spherical and devoid of any cracks and crevices (Fig. 1). The shapeof a nanoparticle is also important for good drug loading. Smooth and regular surface of particles is required for good drug loading. Irregular, cracked surfaced particles often cause leaching of the drug which hampers their release properties. A smooth and spherical surfaced particle also imparts to good flow properties (Venkatesan et al., 2012). The size of the formed nanoparticles can be controlled by managing the rate of addition of the organic phase into the aqueous phase. It can be observed that an increase in the rate of mixing of the two phases leads to a decrease in the particle size, as well as in its entrapment efficiency. The average particle size ofdiclofenac nanoparticle (Fig. 2) was found to 
Table 3. Observations of $\%$ encapsulation efficiency and $\%$ drug release.

\begin{tabular}{llll}
\hline Batch & $\begin{array}{l}\text { \% Encapsulation } \\
\text { efficiency }\end{array}$ & $\begin{array}{l}\text { \% Drug } \\
\text { loading }\end{array}$ & $\begin{array}{l}\text { \% Drug } \\
\text { release }\end{array}$ \\
\hline F1 & 86.34 & 29.12 & 93.23 \\
\hline F2 & 99.03 & 35.32 & 99.03 \\
\hline F3 & 86.45 & 26.98 & 86.45 \\
\hline F4 & 78.98 & 22.19 & 78.98 \\
\hline F5 & 89.34 & 24.98 & 84.45 \\
\hline F6 & 79.99 & 10.34 & 73.08 \\
\hline F7 & 77.45 & 20.78 & 77.45 \\
\hline F8 & 73.45 & 15.23 & 73.45 \\
\hline F9 & 75.45 & 18.12 & 75.45 \\
\hline
\end{tabular}

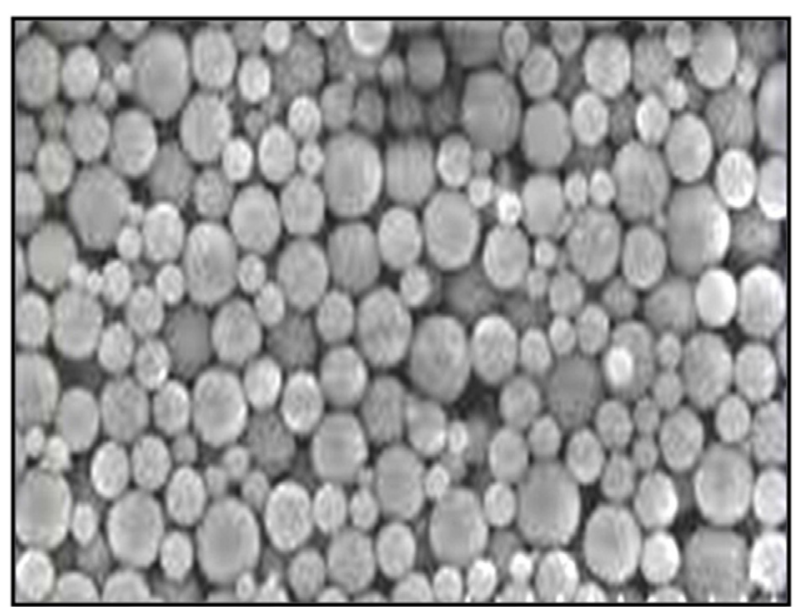

Figure 1. SEM image of diclofenac nanoparticle showing regularly shaped particles.

range between $87 \pm 0.47$ to $103 \pm 0.26 \mathrm{~nm}$. Determination and control of particle size in formulation are important parameters as it directly affects the drug release rate. Larger surface area will be made available for smaller particles so the drug can be loaded into them and be exposed to the particle surface. This will lead to faster release of the drug. If larger particles will be present, then the particles will tend to aggregate during their storage and transportation. Hence, it can be said that the smaller the particle size, the higher stability of the system. Some studies have shown a relationship between the rate of polymeric degradation and particle size, where degradation rate increases with larger particles (Venkatesan et al., 2011).

Azeta potential of the prepared nanoparticles was found to be in the range of $0.598 \pm 0.34$ to $0.826 \pm 0.5$ $\mathrm{mV}$. Electrostatic interactions of nanoparticles with either biological environment or bioactive compounds is largely manipulated by nature and intensity of charge present on the surface. This colloidal stability can be measured using Zeta potential (potential difference between outer plane to surface shear), viz an indirect measure of surface charge present on particles. The knowledge of this value is important, as it can predict storage stability of the systems and is simply the indication of attractive and repulsive forces between particles. For a stable system to form, the values of zeta potential must be to the higher side (either positive or negative). The

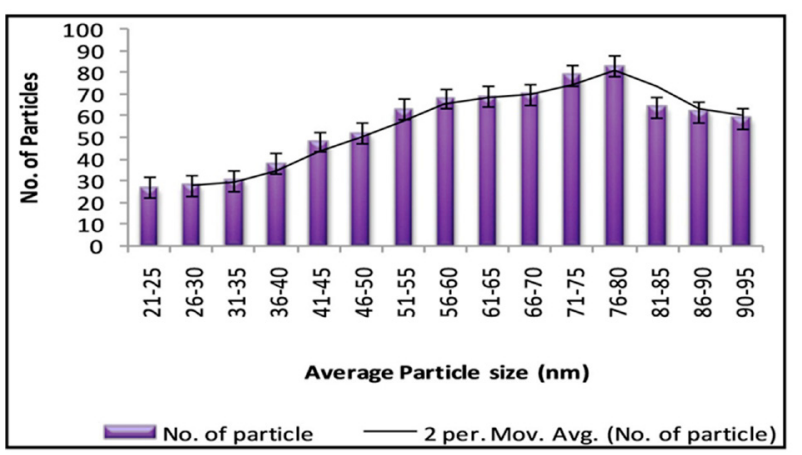

Figure 2. Particle size distribution for diclofenac nanoparticles.

higher the zeta potential, the lesser formation of particle aggregates and the higher system stability (Pangi et al., 2003).

Particle size distribution of nanoparticle can be seen in Fig. 2. It can be seen that the average particle size was found to be $79 \mathrm{~nm}$, while a large number of particles was in the rage of 76-80 $\mathrm{nm}$. By definition, the encapsulation efficiency is the ratio of experimental percentage drug content to that of actual or theoretical outcome, and mostly depends on the nature and concentration of polymers used. Hydrophobic polymers encapsulate large amounts of hydrophobic drugs, while hydrophilic drugs are encapsulated by hydrophilic polymers.

The results obtained showed that with increasing concentration of the polymer, the $\% \mathrm{EE}$ also increases ( $\mathrm{Ta}-$ ble 3).

From Table 3 it can be seen that as the ratio of the polymer and the surfactant increases, the $\%$ encapsulation efficiency also increases. From among all the batches formulated, the F2 batch showed the highest encapsulation efficiency and \% drug release. The biggest advantage of nanotechnology is capability to deliver drugs at targeted sites at a predetermined rate for comparatively long periods of time. The prime requirement is that the drug and its vehicle must be separated. Drug loading can be defined as the amount of drug bound per mass of polymer. Different spectrophotometric techniques such as UV, HPLC, and gel filtration can be used to quantify the amount of drug loaded. The maximum \% drug loading for diclofenac nanoparticles was found to be $35.32 \%$ for formulation batch F2 (Table 3). The reason underlying this observation can be stated as the use of encapsulating polymer. The higher the amount of polymer used, the higher drug loading is possible. From Table 3 it can

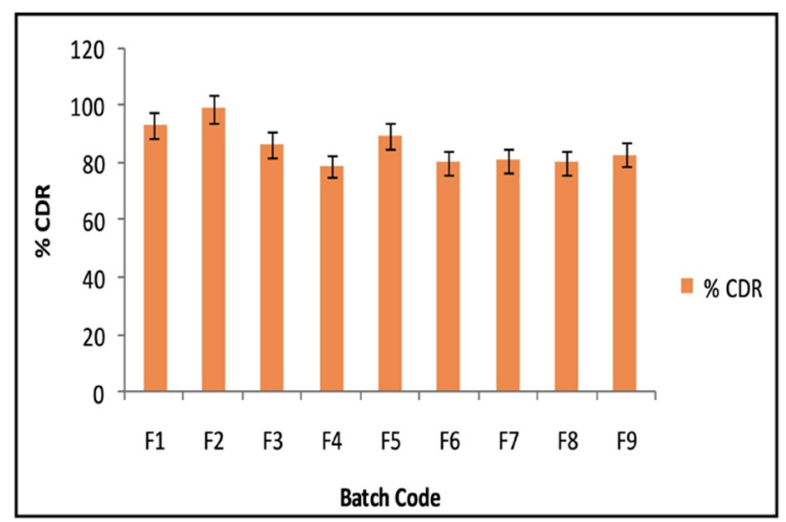

Figure 3. In-vitro drug release from diclofenac nanoparticles as $\%$ cumulative drug release from all batches of formulation. 


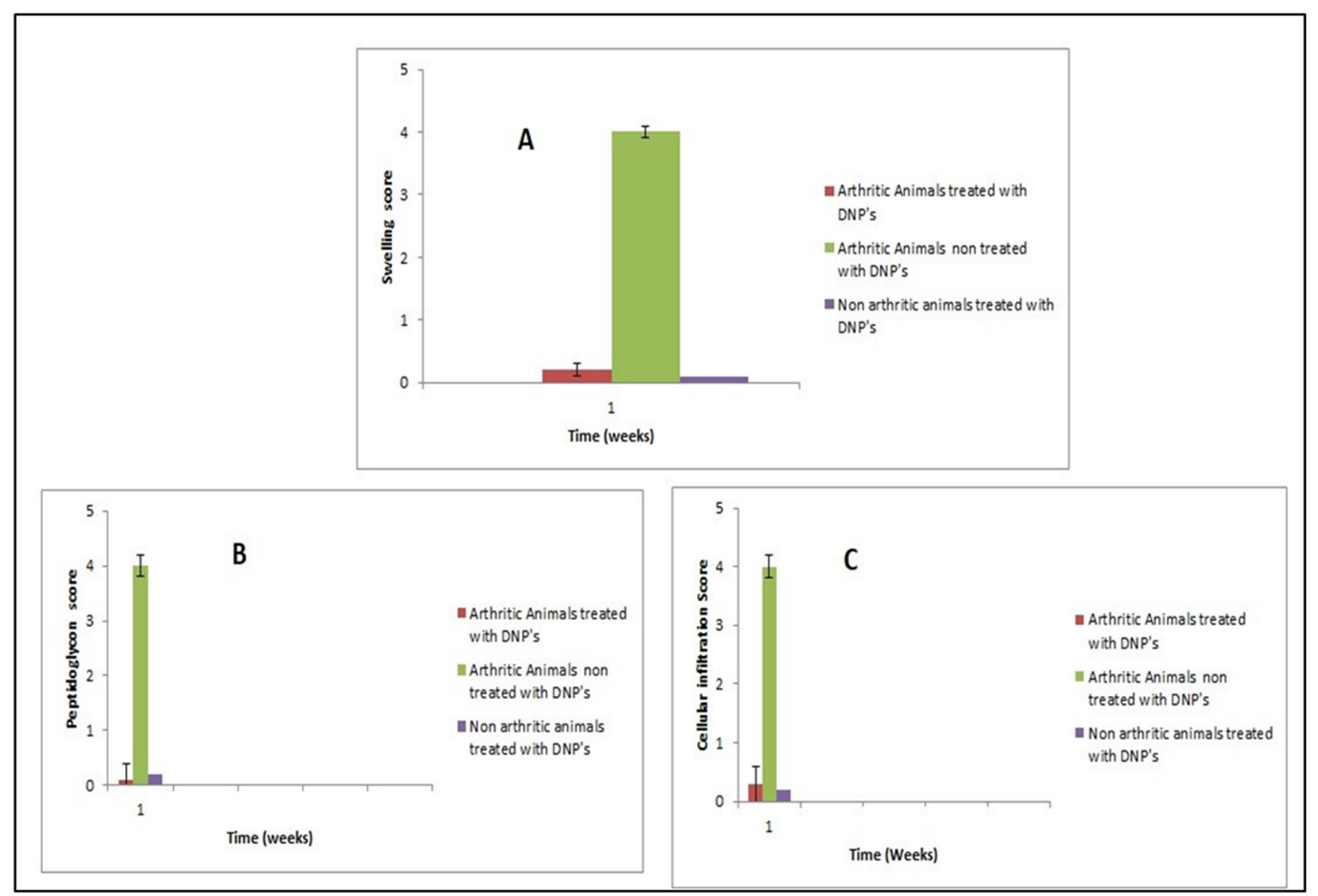

Figure 4. (A) Swelling score; (B) proteoglycan score and (C) cellular infiltration score in three different types of animal models.

be seen that as concentration of the polymer decreases, the \% drug loading also declines (Lu et al., 1999). This loading efficiency can be increased by making a reasonable amount of polymer available for drug entrapment. Sometimes, hydrophilic drugs can encounter problems in this situation.

Theinvitro drug release study was performed using a dialysis membrane. Phosphate buffer solution, $\mathrm{pH} 7.5$, was used as a dissolution media so as to evaluate the dissolution pattern. From all of the batches formulated, F2 was found to be superior in releasing the drug at a sustained manner for longer periods of time. The F2 formulation showed initial burst release of $24.21 \%$, followed by sustained release of diclofenac till $99.03 \%$ up to $12 \mathrm{hrs}$ (Fig. 3). The initial burst release serves as a loading dose, which helps to reach a desired plasma concentration in the system and later the sustained release pattern helps to maintain that level. The reason for this may be the unentrapped drug particles adsorbed on the surface of nanoparticles. When these come in contact with the solvent, they tend to be releasedimmediately into solution to give burst release, then after degradation and/ or erosion of polymeric membrane, the drug particles embedded in the polymer tend to be released.

Assessment of arthritis was carried out after sacrificing the test animals. The joints were decalcified with $5 \%$ formic acid. Sections of bone, cartilage and synovium were prepared and stained with hematoxylin and eosin

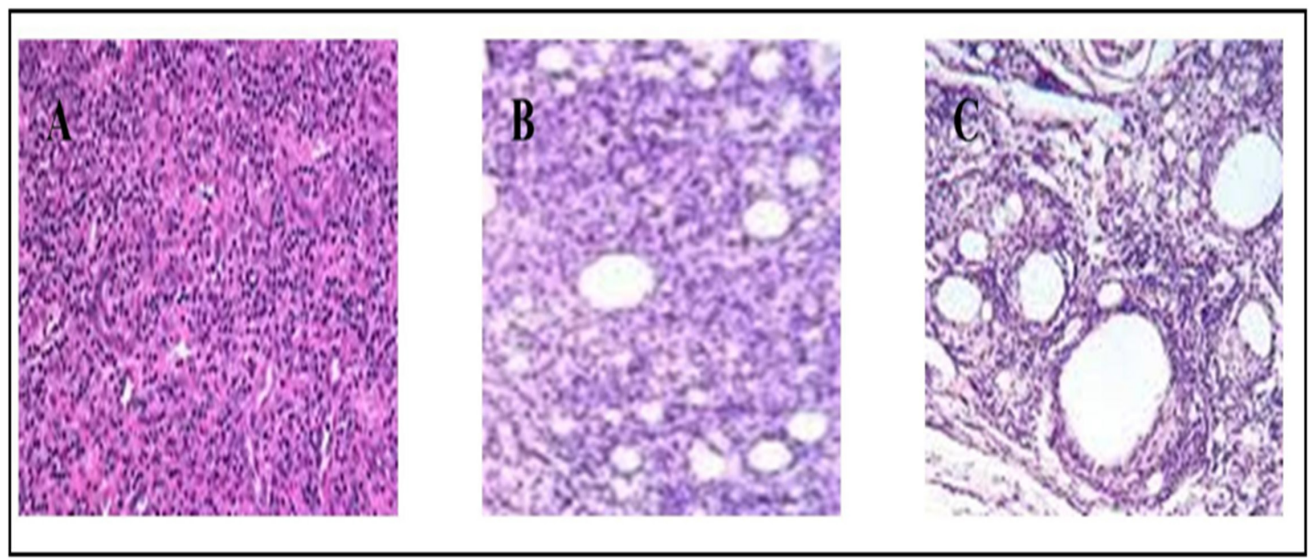

Figure 5. (A) Histopathology of arthritic animals treated with diclofenac nanoparticles; (B) arthritic animals not treated with diclofenac nanoparticles; (C) non-arthritic animals treated with diclofenac nanoparticles.

Treatment group: arthritic animals treated with diclofenac nanoparticles $(\mathrm{N}=7)$, positive control group: arthritic animals not treated with diclofenac nanoparticles $(N=7)$, negative control: non-arthritic animals treated with diclofenac nanoparticles $(N=7)$. 
for proteoglycan content. Synovial inflammation and cartilage degradation were evaluated by blinded histological evaluation of parapatellar synovium and femoral condylar articular cartilage, respectively. The grading of cellular infiltration and swelling was scored from $0-4$ depending on erythema, swelling and cellular infiltration ( 0 , normal; 4, maximum). The score for proteoglycan loss was set from 0 (normal) to 4 (almost total loss of stained proteoglycans) (Laan et al., 1999). See Fig. 4.

The arthritis assessment in preclinical stage was performed in 25 animals which were subjected for induction of arthritis. After performing procedures,a total of 16 animals were found to be arthritic, while 4 were found to be non arthritic. To carry out preclinical evaluation, these animals were divided into three different groups. The group of 16 animals was divided into two groups referred to as a treatment group and a negative control group. The remaining animals were placed in a positive control group. Histopathology of animal tissues is shown in Fig. 5.

After performing the intra-articular injection (optimized batch F2) into animals, they were sacrificed after period of one week. The swelling score was observed and noted. The swelling was found to be higher in case of the positive control group where no administration of diclofenac nanoparticles was done, while the treatment group showed a significant reduction in swelling when compared to the treatment group. Animals with arthritis can be identified by a significant reduction in proteoglycan loss. The positive control group which did not receive treatment showed a significant amount of proteoglycan loss (Bouysset et al., 2006). The normal histological appearance can be observed in Fig. 4A, which shows a continuous layer of cartilage (Score 0), while Fig. 4B explains arthritic knee treated with diclofenac nanoparticles with slight loss of proteoglycan top layer, while a heavy loss of proteoglycan layer can be observed in Fig. 4C for positive control group with no treatment received. The results obtained above prove the efficacy of diclofenac nanoparticles in treating arthritis.

\section{CONCLUSION}

The study presented here highlights a successful formulation of diclofenac nanoparticles for intraarticulate injection to treat arthritis in experimental rabbits. The formulation was found to be suited in all aspects to be delivered intra-particularly. Efficacy studies performed emphasize on its effectiveness in hindering few underlying causes of arthritic conditions. High blood concentrations can be attained by delivering diclofenac nanoparticle via the intraarticular route with subsequent reduction in the dosing frequency.

\section{Conflict of Interest}

The author(s) declare(s) that there is no conflict of interest regarding publication of this paper.

\section{REFERNCES}

Armstrong RD, English J, Gibson T (1981) Serum methylprednisolone levels following intra-articular injection of methyl prednisolone acetate. Ann. Rheum. Dis. 40: 571-574. https://doi.org/10.1136/ ard.40.6.571

Bouysset M, Hugueny P, Gintz B (2006) Corticosteroid injections and synoviortheses of the foot and ankle in rheumatoid arthritis. Foot and ankle in rheumatoid arthritis. Paris: Springer-Verlag. 120

Couvreur P, Dubernet C, Puisieux F (1995) Controlled drug delivery with nano particles:current possibilities and future trends. Eur. J. Pharm. Biopharm. 41: 2-13.

Dong RK, Gi YP (2012) Intraarticular Injections for the treatment of osteoarthritis: Focus on the clinical use of several regimens. INTECH Open Access Publisher. 2: 67-100. https://doi. org/10.5772/26396

Egemen A, Hayrettin K, Isik A (2014) Intraarticular injections (corticosteroid, hyaluronic acid, platelet rich plasma) for the knee osteoarthritis. World J. Orthop. 5: 351-361. https://doi.org/10.5312/wjo. v5.13.351

Gibofsky A (2012) Overview of epidemiology, pathophysiology, and diagnosis of rheumatoid arthritis. Am J. Manag. Care 18 (Suppl): S295

Gibofsky A (2014) Epidemiology, pathophysiology, and diagnosis of rheumatoid arthritis: A Synopsis. Am J. Manag. Care 20 (Suppl 7): S128-S135

Karuppusamy C, Venkatesan P (2017) Role of nanoparticles in drug delivery system: A comprehensive review. J. Pharm. Sci. Res. 9: 318325

Kim S, Hwang J, Xuan J, Jung YH, Cha HS, Kim KH (2014) Global metabolite profiling of synovial fluid for the specific diagnosis of rheumatoid arthritis from other inflammatory arthritis. PloS One 2; 9(6): e97501. https://doi.org/10.1371/journal.pone.0097501

Laan RF, Jansen TL, Van Riel PL (1999) Glucocorticosteroids in the management of rheumatoid arthritis. Rheumatology (Oxford) 38: 6-12. https://doi.org/10.1093/rheumatology/38.1.6

Liao AH, Chung HY, Chen WS, Yeh MK (2016) Efficacy of Combined ultrasound-and-microbubbles-mediated diclofenac gel delivery to enhance transdermal permeation in adjuvant-induced rheumatoid arthritis in the rat. Ultrasound Med. Biol. 42: 1976-1985. https://doi. org/10.1016/j.ultrasmedbio.2016.03.031

Lu JX, Prudhommeaux A, Meunier LS, Guillemin G (1999) Effects of chitosan on rat knee cartilages. Biomaterials 20: 1937-1944. https:// doi.org/10.1016/s0142-9612(99)00097-6

Malmstrom V, Catrina AI, Klareskog L (2017) The immunopathogenesis of seropositive rheumatoid arthritis: from triggering to targeting. Nat. Rev. Immunol. 17: 60-75. https://doi.org/10.1038/nri.2016.124

Mirajkar R, Kurkute R, Madgulkar A (2018) Formulation development of sustained release intra-articular injection of analgesic drug. J. Drug Deliv. Therap. 8: 209-217. https://doi.org/10.22270/jddt.v8i4.1770

Nissen SE, Yeomans ND, Solomon DH, Lüscher TF, Libby P, Husni ME, Graham DY, Borer JS, Wisniewski LM, Wolski KE, Wang Q (2016) Cardiovascular safety of celecoxib, naproxen, or ibuprofen for arthritis. N. Engl. J. Med. 375: 2519-2529. https://doi. org/10.1056/NEJMoa1611593

Pangi Z, Beletsi A, Evangelatos K (2003) PEG-ylated nanoparticles for biological and pharmaceutical application. Adv. Drug. Del. Rev. 55: 403-419. https://doi.org/10.1016/s0169-409x(02)00226-0

Suri SS, Fenniri H, Singh B (2007) Nanotechnology-based drug delivery systems. J. Occup. Med. Toxicol. 2, 16. https://doi.org/10.1186/17456673-2-16

Trouw LA, Mahler M (2012) Closing the serological gap: promising novel biomarkers for the early diagnosis of rheumatoid arthritis. Autoimmun. Rev. 12: 318-322. https://doi.org/10.1016/j.autrev.2012.05.007

Van WA, Pandhi S, Nixon RM, Guyot P, Karabis A, Moore RA (2015) Relative benefit-risk comparing diclofenac to other traditional nonsteroidal anti-inflammatory drugs and cyclooxygenase- 2 inhibitors in patients with osteoarthritis or rheumatoid arthritis: a network metaanalysis. Arthritis. Res. Ther. 17: 66. https://doi.org/10.1186/s13075015-0554-0

Venkatesan P, Janardhanan VS, Muralidharan C, Valliappan K (2012) Improved HPLC method with the aid of chemometric strategy: determination of loxoprofen in pharmaceutical formulation. Acta Chim. Slov. 59: 242-248. PMID: 24061236

Venkatesan P, Manavalan R, Valliappan K (2011) Preparation and evaluation of sustained release loxoprofen loaded microspheres. J. Basic Clin. Pharm. 2: 159-162. PMID: 24826017

Zhang Y, Cun D, Kong X, Fang L (2014) Design and evaluation of a novel transdermal patch containing diclofenac and teriflunomide for rheumatoid arthritis therapy. Asian J. Pharma. Sci. 9: 251-259. https://doi.org/10.1016/j.ajps.2014.07.007 